\title{
Editorial
}

\section{How Well Are We Taking Care of Melanoma Patients in the USA?}

\author{
Vernon K. Sondak, $\mathrm{MD}^{1,2}$ \\ ${ }^{1}$ Division of Cutaneous Oncology, H. Lee Moffitt Cancer Center, 12902 Magnolia Drive, Tampa, FL 33612, USA \\ ${ }^{2}$ Departments of Oncologic Sciences and Surgery, University of South Florida College of Medicine, Tampa, FL, USA
}

Increasingly, "compliance" with adopted standards of practice is becoming synonymous with the delivery of "quality" medical care to our patients. For our melanoma patients, the guidelines promulgated by the National Cancer Centers Networks $(\mathrm{NCCN})^{1}$ are one such set of standards, and have been widely disseminated. But to what extent are we complying with the NCCN melanoma guidelines, and to what extent does compliance with them indicate that highquality medical care is being delivered? Current and recent publications in Annals of Surgical Oncology shed light on these issues, but raise as many questions as they answer.

Earlier this year, Erickson et al. $^{2}$ reviewed the charts of 252 clinically node-negative melanoma patients treated at a community teaching hospital and found a significant rate of noncompliance with NCCN guidelines. Excision margins conformed to the guideline standards in $87 \%$ of in situ and thin (T1) tumors but only $60 \%$ of thicker tumors (T2-4). Compliance with the recommendation for lymph node staging by sentinel node biopsy was noted in only $47-74 \%$ of patients with T2-4 melanomas. Treatment was more likely to be in compliance with guidelines if delivered by a surgical oncologist, and if delivered to a patient younger than age 80 years. The clinical significance of noncompliance, however, was not addressed in this study.

Now, the same group has extended these observations and correlated compliance with oncologic outcomes and complication rates. ${ }^{3}$ In a retrospective

Published online July 9, 2008.

Address correspondence and reprint requests to: Vernon $\mathrm{K}$. Sondak, MD; E-mail: vernon.sondak@moffitt.org

Published by Springer Science+Business Media, LLC $\odot 2008$ The Society of Surgical Oncology, Inc. review of tumor registry data and clinical charts from 327 clinically node-negative melanoma patients treated at the same institution as in the prior study, postoperative complications were 3.4-fold higher for patients treated with surgical margins not in compliance with NCCN guidelines, and 2.4-fold higher for patients whose lymph nodes were managed in a fashion not in compliance with the guidelines (mostly by not performing sentinel node biopsy when indicated in the guidelines or by not performing completion node dissection when the sentinel node was positive). Even more concerning, after a median follow-up of 51 months, noncompliant treatment was associated with locoregional recurrence $(24 \%$ for margin noncompliant treatment versus $6 \%$ for margin compliant treatment and $33 \%$ for lymph node noncompliant treatment versus $6 \%$ for lymph node compliant treatment, both $P<0.01$ ). The age of patients experiencing or not experiencing postoperative complications or locoregional recurrence was not significantly different.

There is also evidence that the significant rates of noncompliance noted in the June issue one hospital represent a pattern of noncompliance that can be seen nationwide. Bilimoria et al., in this issue of Annals of Surgical Oncology, ${ }^{4}$ present an assessment of practice patterns for patients with a positive sentinel lymph node, based on analysis of data in the National Cancer Database, a joint program of the Commission on Cancer and the American Cancer Society. They found that an astonishing $50 \%$ of the 2,942 melanoma patients in the database with positive sentinel nodes did not undergo completion node dissection, as called for in the NCCN guidelines, during 2004-2005. Patients older than 75 years and those with lowerextremity primaries (i.e., those requiring inguinal 
node dissection), were significantly less likely to be treated with completion node dissection after a positive sentinel node biopsy. Other findings of note were that $16.4 \%$ of patients who did not have a positive sentinel node underwent complete node dissection, and that $58.2 \%$ of patients had the sentinel node biopsy and the completion node dissection performed at the same procedure, rather than as two separate surgeries. Care at an NCCN-participant institution or an NCI-designated cancer center was associated with higher rates of completion node dissection $(60.2 \%$ versus $43.8-46.7 \%$ at other types of institutions, $P<0.0001)$, and a greater likelihood of undergoing a two-stage procedure $(52.5 \%$ versus $33.6-43.6 \%, P=0.02)$. No outcome or complication data were provided, however.

So what does this all mean? First of all, it must be acknowledged that the compliance rates and practice patterns described in these three studies absolutely need to be validated. Frankly, the percentages of completion and one-stage lymphadenectomies described in the Bilimoria article at NCCN/NCI cancer centers are not in keeping with the experience of this editorialist, who is familiar with the practice patterns of melanoma surgical oncologists at many of those centers. I am simply unaware of any cancer center in the country today where $40 \%$ of sentinel node-positive patients are treated with nodal observation rather than surgery even now, 3 years after the study (which was conducted prior to the initiation of the Multicenter Selective Lymphadenectomy Trial [MSLT] 2, which offers randomization of sentinel node-positive patients to a nonsurgical arm). The same can be said for a nearly $50 \%$ rate of converting a sentinel node biopsy for melanoma to a completion dissection, based presumably on some type of intraoperative identification of metastatic involvement. Therefore, validation of the data needs to take place, and if systematic errors have crept into the melanoma data captured by the National Cancer Database, these errors should be identified and corrected. However, having said that, there is no doubt that many melanoma patients with positive sentinel nodes are not undergoing completion node dissection, whether based on surgeon recommendation or personal choice, and there is also no doubt that we as melanoma surgeons have no way to say with certainty that such deviation from accepted guidelines is in our patients' best interests long term.

Clearly, when patients with positive sentinel nodes choose to forego complete lymphadenectomy, they are motivated predominantly by a fear of complications, most especially lymphedema. So the findings of
Foster et al. that patients whose lymph node basin was treated in a noncompliant fashion were actually more likely to suffer complications is remarkable and potentially highly significant, although we still need to know more about just what complications these patients experienced. Is it actually credible to think that there would be more complications, and perhaps more lymphedema, for sentinel node-positive patients treated with nodal observation rather than complete dissection? Absolutely!

For melanoma patients with a properly performed sentinel node biopsy treated with a subsequent completion lymph node dissection, long-term lymphedema rates should be low, and prompt and aggressive management of even minimal lymphedema should be instituted to decrease the likelihood that the edema will be life-limiting. Sabel et al. have shown that inguinal node dissection performed for sentinel nodepositive disease is associated with significantly less edema than when the procedure is performed for palpable metastasis. ${ }^{5}$ They also found that patients undergoing inguinal node dissection for palpable metastasis were more likely to have four or more involved nodes or extracapsular extension of the nodal metastasis. The results presented to date of the randomized MSLT-1 trial show a similar finding: patients randomized to nodal observation without sentinel node biopsy who recurred in their nodal basin were significantly more likely to have four or more involved nodes. ${ }^{6}$ Since both involvement of four or more nodes and the presence of extracapsular extension have been identified as risk factors for regional recurrence, postoperative radiation is often utilized in these patients, potentially further increasing the likelihood of significant lymphedema. Patients with recurrent disease in the inguinal basin after a positive sentinel node biopsy may also be more likely to require pelvic (ilioinguinal) node dissection to achieve regional control; the potential impact of that on long-term morbidity needs to be addressed.

But what of all those sentinel node-patients who undergo completion node dissection only to be told that all the harvested nodes were negative - especially if the sentinel node itself contained only small foci of disease seen on immunohistochemistry? Does this really represent "prognostic false positivity" as we have heard over and over lately despite the absence of supportive data. ${ }^{7-9}$ A number of points need to be made in this regard. First and foremost, completion node dissection specimens are rarely if ever analyzed with the same techniques - serial sectioning and immunohistochemistry - that were used to identify the positive sentinel node in the first place. So it 
should be no surprise that the remaining nodes are "negative" when the index node, the sentinel node, may well have been considered "negative" if analyzed the same way. Second, the already cited discrepancy seen in the MSLT-1 randomized trial between the number of involved nodes found at the time of completion dissection after sentinel node biopsy and the number of involved nodes found in patients randomized to nodal observation should remind us all that nodes initially classified histopathologically as negative can later be found to contain tumor if left in place. Thirdly, we must remember that melanoma metastases in regional lymph nodes can take years and even decades to become clinically evident. Studies showing that nodal micrometastases have little or no therapeutic significance at 5 years or less of follow-up may merely be telling us that small tumors take longer to kill patients than large tumors, not that they lack the ability to kill them. ${ }^{10}$

Finally, we need to examine a widely held but unproven tenet that drives much of the noncompliant management of lymph node basins: more favorable disease is more easily cured with less treatment. The available data, augmented now by the cautionary results of Foster et al. and new studies suggesting that adjuvant interferon offers the most benefit to patients with the least burden of nodal metastases, ${ }^{11,12}$ are also compatible with the alternative viewpoint that most of the therapeutic value of aggressive surgical management and systemic therapy for stage III melanoma is confined to those patients who present with the smallest burden of disease and/or the fewest initially involved nodes. If we forego aggressive treatment for these patients, we risk missing the very narrow window available to us for cure in this disease.

So where do we go from here? Further studies of compliance with melanoma treatment guidelines and the therapeutic impact of noncompliance are clearly necessary. On the other hand, there are certainly good reasons why specific patients might be best served by individualized or personalized treatment not suited to the majority of cases. Further research in personalized management is also clearly necessary, including an improved understanding of which factors besides chronologic age are most associated with surgical complications and poor oncologic outcomes in our expanding cohort of elderly patients with melanoma. It is no longer sufficient to say that all patients over a certain age should be treated differently than their younger counterparts. However, as melanoma surgeons we bear an additional burden, one that we have in large part neglected, and that is to minimize the morbidity of the curative treatments we have for melanoma while not sacrificing the benefits. Appropriately but not excessively wide margins of excision, oriented properly particularly on the extremity, achieve excellent local control rates with modest morbidity - but we can still do better. ${ }^{13}$ Nowhere is this more evident than in the management of the inguinal and pelvic lymph nodes, where minimizing long-term morbidity while maximizing regional control should become the goal of every melanoma surgeon and the focus of significant research, ${ }^{14}$ so that someday every melanoma patient will be able to proceed with therapy confident that compliance with recommended guidelines represents their very best chance for cure and for optimum quality of life.

\section{REFERENCES}

1. National Comprehensive Cancer Network. NCCN clinical practice guidelines in oncology. Melanoma 2008. Accessed at www.ncen.org.

2. Erickson JL, Velasco JM, Hieken TJ. Compliance with melanoma treatment guidelines in a community teaching hospital: time trends and other variables. Ann Surg Oncol 2008; 15:1211-7.

3. Foster JE, Velasco JM, Hieken TJ. Adverse outcomes associated with non-compliance with melanoma treatment guidelines. Ann Surg Oncol 2008; DOI: 10.1245/s10434-008-0021-0 [ASO-2008-03-0221].

4. Bilimoria KY, Balch CM, Bentrem, et al. Complete lymph node dissection for sentinel node-positive melanoma: assessment of practice patterns in the United States. Ann Surg Oncol 2008; 15(6):1566-76; [ASo-2008-01-0011].

5. Sabel MS, Griffith KA, Arora A, et al. Inguinal node dissection for melanoma in the era of sentinel node biopsy. Surgery 2007; 141:728-35.

6. Morton DL, Thompson JF, Cochran AJ, et al. Sentinel-node biopsy or nodal observation in melanoma. $N$ Engl J Med 2006; 355:1307-17.

7. Thomas JM. Prognositic false-positivity of the sentinel node in melanoma. Nat Clin Pract Oncol 2008; 5:18-23.

8. Thomas JM, Hayes AJ. Sentinel lymph node biopsy in melanoma in 2008. PPO Updates 2008; 22:1-9.

9. Thomas JM. Sentinel lymph node biopsy in malignant melanoma. Brit Med $J$ 2008; 336:902-3.

10. VanAkkooi ACJ, deWilt JHW, Verhoef C, et al. Isolated tumor cells and long-term prognosis of patients with melanoma. Ann Surg Oncol 2008; 15(5):1547-8.

11. Eggermont AM, Suciu S, Santinami M, et al. EORTC 18991: Long-term adjuvant pegylated interferon-alpha2b (PEG-IFN) compared to observation in resected stage III melanoma, final results of a randomized phase III trial. J Clin Oncol 2007; 25:473s.

12. Anaya DA, Xing Y, Feng L, et al. Adjuvant high-dose interferon for cutaneous melanoma is most beneficial for patients early stage III disease. Cancer 2008; 112:2030-7.

13. Riker AI, Sondak VK. (2006) Complications of soft-tissue tumor surgery. In: Mulholland MW, Doherty GM (eds). Complications in surgery. Lippincott Williams \& Wilkins, Philadelphia, PA. pp 619-27.

14. Sarnaik AA, Puleo CA, Sondak VK. Limiting the morbidity of inguinal lymphadenectomy for metastatic melanoma. Cancer Control $J$ (in press). 